\title{
Oxidation Induced Doping of Nanoparticles Revealed by in Situ X-ray Absorption Studies
}

\author{
Soon Gu Kwon, ${ }^{\dagger}$ Soma Chattopadhyay, ${ }^{\ddagger}, \nabla$ Bonil Koo, ${ }^{\dagger}$ Paula Cecilia dos Santos Claro, ${ }^{\dagger, \S}$ \\ Tomohiro Shibata, ${ }^{\ddagger \nabla}$ Félix G. Requejo, ${ }^{\S}$ Lisandro J. Giovanetti, ${ }^{\S}$ Yuzi Liu, ${ }^{\dagger}$ Christopher Johnson, \\ Vitali Prakapenka, ${ }^{\perp}$ Byeongdu Lee, ${ }^{\#}$ and Elena V. Shevchenko*, ${ }^{\dagger}$ \\ ${ }^{\dagger}$ Nanoscience and Technology Division, "Chemical Science \& Engineering Division, and "Advanced Photon Source, Argonne \\ National Laboratory, 9700 South Cass Avenue, Argonne, Illinois 60439, United States \\ ${ }^{\ddagger}$ CSRRI-IIT, MRCAT, Argonne National Laboratory, Argonne, Illinois 60439 United States \\ $\nabla$ Physics Department, Advanced Materials Group, Illinois Institute of Technology, Chicago, Illinois 60616, United States \\ ${ }^{\S}$ Instituto de Investigaciones Fisicoquímicas Teóricas y Aplicadas (INIFTA), Dpto. de Química, FCE-UNLP, CONICET, La Plata \\ 1900 Argentina \\ ${ }^{\perp}$ Center of Advanced Radiation Sources, University of Chicago, Argonne, Illinois 60439, United States
}

\section{Supporting Information}

\begin{abstract}
Doping is a well-known approach to modulate the electronic and optical properties of nanoparticles (NPs). However, doping at nanoscale is still very challenging, and the reasons for that are not well understood. We studied the formation and doping process of iron and iron oxide NPs in real time by in situ synchrotron X-ray absorption spectroscopy. Our study revealed that the mass flow of the iron triggered by oxidation is responsible for the internalization of the dopant (molybdenum) adsorbed at the surface of the host iron NPs. The oxidation induced doping allows controlling the doping levels by varying the amount of dopant precursor. Our in situ studies also revealed that the dopant precursor substantially

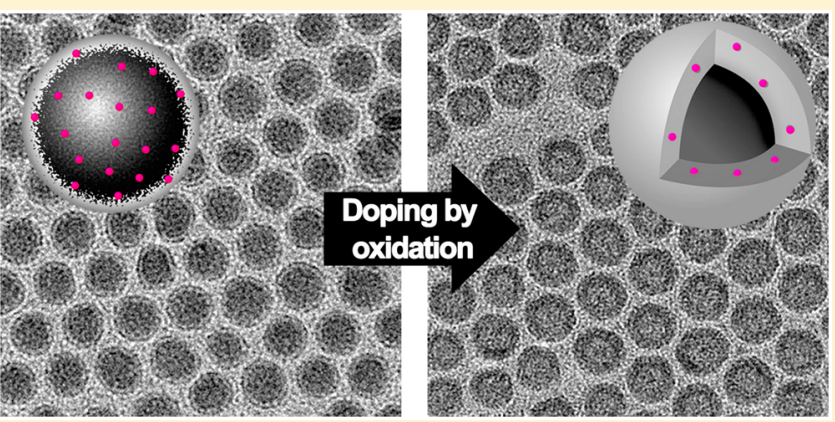
changes the reaction kinetics of formation of iron and iron oxide NPs. Thus, in the presence of dopant precursor we observed significantly faster decomposition rate of iron precursors and substantially higher stability of iron NPs against oxidation. The same doping mechanism and higher stability of host metal NPs against oxidation was observed for cobalt-based systems. Since the internalization of the adsorbed dopant at the surface of the host NPs is driven by the mass transport of the host, this mechanism can be potentially applied to introduce dopants into different oxidized forms of metal and metal alloy NPs providing the extra degree of compositional control in material design.
\end{abstract}

KEYWORDS: Doping, metal oxide, Kirkendall effect, hollow nanoparticles, in situ studies

$\mathrm{D}$ oping has led to a revolutionary breakthrough in the design of semicondutor devices. Yet, it is still challenging to introduce dopants in nanoparticles (NPs) in order to access properties unavailable in the undoped materials. ${ }^{1-3}$ Different types of interactions between host and dopant are responsible for the properties of doped NPs. The new properties in doped NPs may originate from spin exchange interactions between the dopant and host, ${ }^{4-6}$ presence of long-lived highly isolated electronic states, ${ }^{7,8}$ or simply from high concentration of structural defects. ${ }^{9}$ Recently, it has been demonstrated that doping of bulk $\gamma-\mathrm{Fe}_{2} \mathrm{O}_{3}$, that is traditionally considered as an anode material in lithium-ion batteries, ${ }^{10}$ with high-valent metal cations such as $\mathrm{Mo}^{6+}$ results in more than five-times increase in $\mathrm{Li}^{+}$ion capacity in the cathode range from 4.1 to $2.0 \mathrm{~V}$ (vs $\mathrm{Li}$ / $\left.\mathrm{Li}^{+}\right) .{ }^{11,12}$ Charge compensation in $\mathrm{Mo}^{6+}$-doped $\gamma-\mathrm{Fe}_{2} \mathrm{O}_{3}$ assumes the formation of an extra cation vacancy $(\square)$ per each $\mathrm{Mo}^{6+}\left[\mathrm{Fe}_{2} \mathrm{O}_{3}+x \mathrm{Mo}^{6+} \rightarrow \mathrm{Fe}_{2(1-x)} \square_{x} \mathrm{Mo}_{x} \mathrm{O}_{3}+2 x \mathrm{Fe}^{3+}\right]$ in iron oxide matrix. As the concentration of cation vacancy increases, metal oxide can accommodate insertion of more $\mathrm{Li}^{+}$ ions leading to higher capacities in the cathode range that tunes $\gamma-\mathrm{Fe}_{2} \mathrm{O}_{3}$ into cathode material.

Despite the growing importance of the doped NPs for a broad range of applications, their synthesis is facing challenges since doping NPs is in general more difficult than their bulk counterpart. ${ }^{5,13-15}$ Difficulty of doping may have thermodynamic nature. Previously, it was suggested that the defect formation energy in NPs is higher than that of bulk host materials, and as a result, the doped NPs are thermodynamically less stable than the undoped ones. ${ }^{14}$ However, with the

Received: March 14, 2016

Revised: April 22, 2016

Published: May 6, 2016 

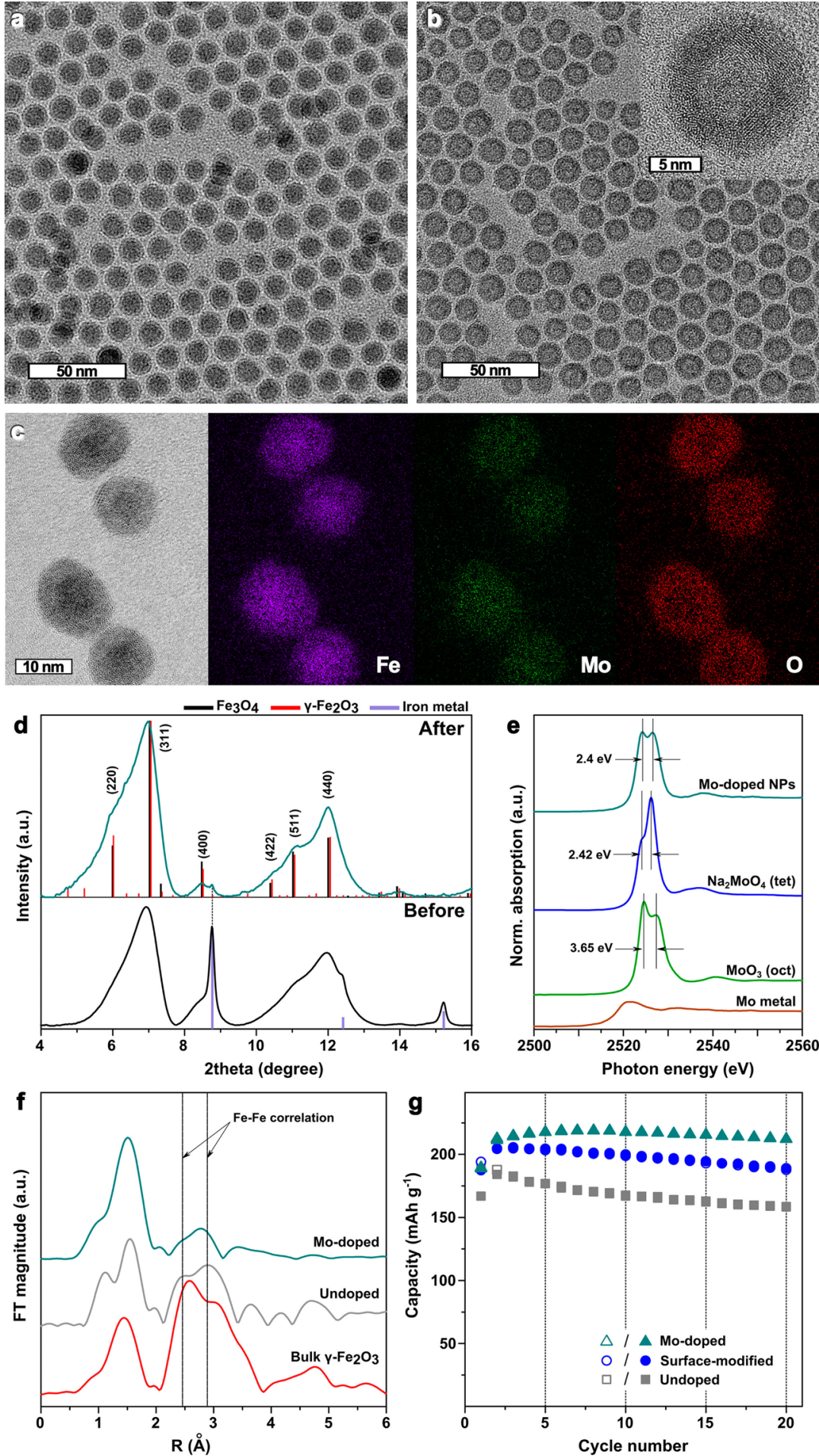

Figure 1. Characterization of Mo-doped hollow iron oxide NPs. (a,b) TEM images of the iron NPs before oxidation (a) and Mo-doped iron oxide NPs after oxidation (b), respectively. The inset in panel $b$ is a high-resolution TEM image of a hollow shell NP. (c) Bright-field and energy-filtered TEM images of iron/iron oxide core/hollow shell NPs. Elemental mapping was performed by filtering at Fe L-edge (purple), Mo M-edge (green), and $\mathrm{O}$ K-edge (red). (d) XRD patterns of the NPs before and after oxidation. (e) XANES spectra on Mo $L_{3}$-edge of Mo-doped hollow iron oxide NPs and the standard Mo species. (f) EXAFS data on Fe K-edge from bulk $\gamma$ - $\mathrm{Fe}_{2} \mathrm{O}_{3}$ and undoped and Mo-doped hollow iron oxide NPs. (g) $\mathrm{Li}^{+}$ion capacity as a function of cycle number for Mo-doped $(\sim 6.37 \%)$ and undoped hollow iron oxide NPs and the hollow iron oxide NPs prepared by surface modification of iron NPs with $\mathrm{MoO}_{2}(\mathrm{acac})_{2}$ followed by subsequent oxidation (such procedure results in $\sim 6 \%$ of Mo). The samples were cycled at $300 \mathrm{~mA} / \mathrm{g}$ in the voltage range of $3.5-1.5 \mathrm{~V}$ (vs $\mathrm{Li} / \mathrm{Li}^{+}$).

progress in the synthetic strategies, it was established that the reaction kinetics also plays a crucial role in synthesis of doped NPs and allows their synthesis under thermodynamically unfavorable conditions. ${ }^{1,13}$ As a result, different synthetic strategies based on control over reaction kinetics via adjustment of the reactivity of the precursors, reaction temperature, and diffusion have been proposed. ${ }^{5,16-21}$ When the dopant and host precursors have similar reactivity, the dopant atoms can 

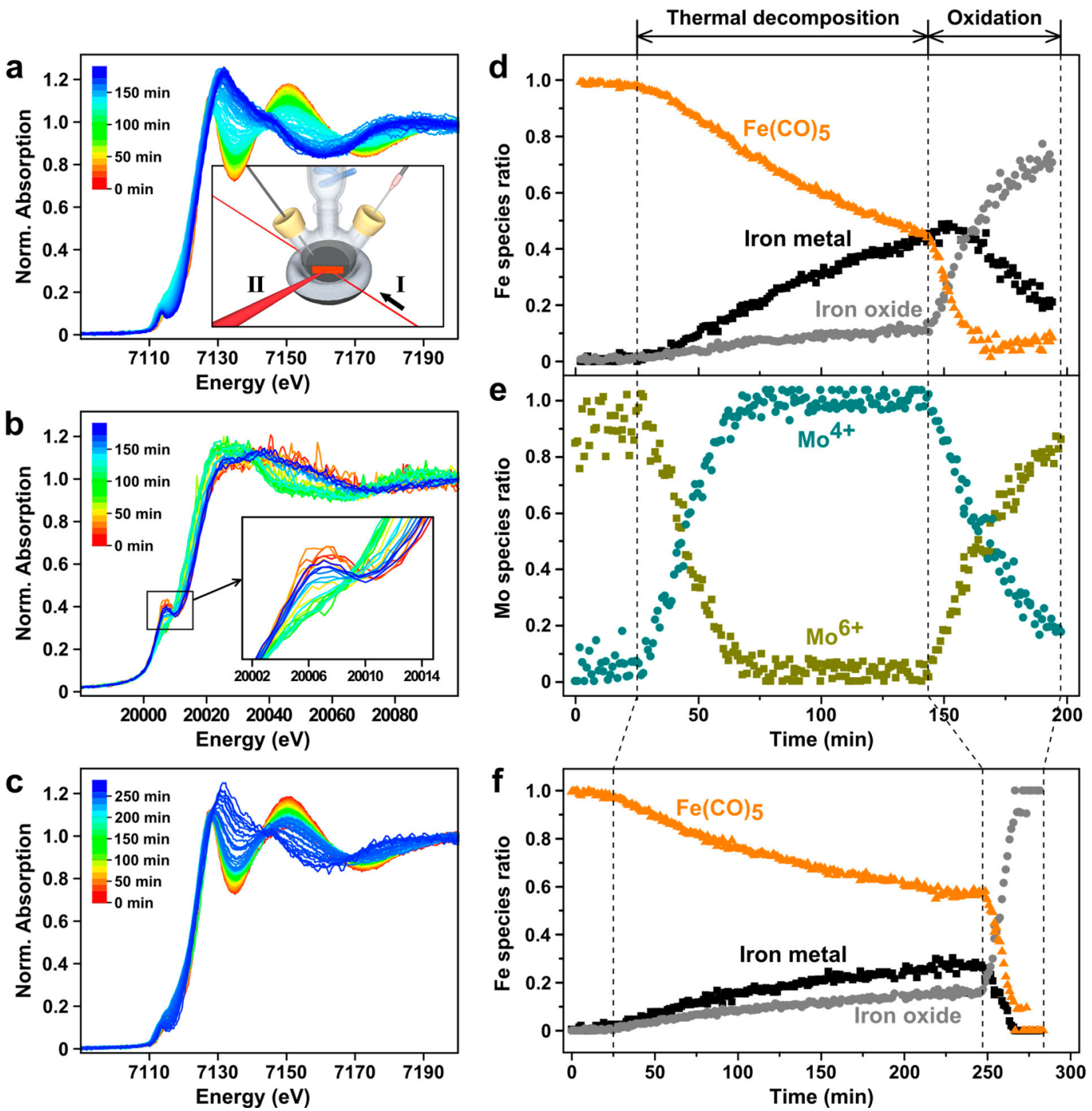

Figure 2. Temporal changes of Fe and Mo XANES spectra and the ratio of iron and molybdenum species during the synthesis of Mo-doped and undoped hollow iron oxide NPs. (a,b) XANES spectra acquired during the synthesis of Mo-doped hollow iron oxide NPs at Fe K-edge (a) and Mo $K$-edge (b). The inset in panel a depicts the measurement setup and the pathways of the incident (I) and fluorescent (II) X-ray beam. The inset in panel $b$ shows the magnified prepeak range. (c) Fe K-edge XANES spectra acquired during the synthesis of undoped hollow iron oxide NPs. (d,e) Temporal change in the ratio of iron and molybdenum species in the reaction mixture as a function of time during the synthesis of Mo-doped hollow iron oxide NPs. The reaction temperature reached $180{ }^{\circ} \mathrm{C}$ at $37 \mathrm{~min}$. (f) Temporal change of iron species ratio during the synthesis of undoped hollow iron oxide NPs. The reaction temperature reached $180^{\circ} \mathrm{C}$ at $32 \mathrm{~min}$. The data in panels $\mathrm{d}$, e, and $\mathrm{f}$ are derived from XANES spectra in panels a, b, and c, respectively.

participate in the nucleation and growth of the host material and direct in situ doping is possible. ${ }^{21}$ When doping of NPs is achieved by trapping of the dopant atoms at the surface of nucleated core, the low reaction temperature is found to prevent the dopant from diffusing out of the NPs during their further growth. ${ }^{22,23}$ Also, doping in NPs can occur as a result of inward diffusion of the dopant from the solution into the lattice of undoped NPs followed by cation exchange reactions between the dopant and host. ${ }^{24,25}$ This method exploits the fact that the ionic diffusion rate in NPs is higher than that in bulk. $^{26}$

Despite a substantial progress in synthesis of doped NPs, our understanding of the doping mechanisms is still mainly based on ex situ techniques including optical spectroscopy, electron paramagnetic resonance (EPR), and transmission electron microscopy (TEM) analyses that provide only limited information on the doping reaction kinetics. ${ }^{21,22,24}$ Successful synthesis of doped NPs requires correlation of various kinetic parameters, and hence, time-resolved studies on the doping processes are critical to resolve the doping mechanism.

Being inspired by the enhanced electrochemical properties of bulk $\gamma$ - $\mathrm{Fe}_{2} \mathrm{O}_{3}$ doped with $\mathrm{Mo}^{6+}$ cations mentioned above, we conjectured that high-valent cation doping of metal oxide NPs, that already have high level cation vacancy, ${ }^{27}$ can be a promising strategy to further increase the $\mathrm{Li}^{+}$ion capacity. ${ }^{11,12}$ Thus, we selected synthesis of molybdenum-dope hollow iron oxide NPs. In order to access the reaction kinetics and to study the doping mechanism, we conducted real-time in situ synchrotron quick-scanning X-ray absorption spectroscopy (XAS) technique that allowed simultaneous monitoring of reaction kinetics of the precursors, compositional change of the NPs, and the chemical state (valency and coordination geometry) and position (whether it is in the solution or in the host lattice) of the dopant. Our time-resolved XAS study on 
the synthesis of molybdenum-doped hollow iron oxide NPs revealed an oxidation induced doping mechanism by which the mass transport of the host (iron and oxygen) induces the internalization of dopant atoms (molybdenum) into the lattice of oxidized NPs $\left(\gamma-\mathrm{Fe}_{2} \mathrm{O}_{3}\right)$. In addition, we discovered that the presence of the dopant (molybdenum species) changes both the decomposition kinetics of the iron precursor and NP oxidation and stabilizes the NPs as inorganic ligands. Moreover, the same phenomena have been also observed for cobalt NP systems.

Synthesis and ex Situ Characterization of Mo-Doped Hollow Iron Oxide NPs. The synthesis of Mo-doped hollow iron oxide NPs was conducted using a modification of the method developed for hollow iron oxide NPs synthesis (see Scheme S1 in Supporting Information)..$^{27,28}$ In a typical reaction, iron pentacarbonyl $\left[\mathrm{Fe}(\mathrm{CO})_{5}\right]$ is introduced into a reaction mixture containing the molybdenum precursor $\left[\mathrm{MoO}_{2}(\mathrm{acac})_{2}\right]$ and oleyamine at elevated temperature that results in the thermal decomposition of iron precursor and formation of metallic NPs (Figure 1a). Then bubbling of dry air into the mixture leads to the formation of Mo-doped hollow iron oxide NPs via Kirkendall effect (Figure 1b). ${ }^{28}$

Energy-filtered TEM images of the half-oxidized NPs that have the iron core and the iron oxide shell show that Mo is evenly distributed within the iron oxide shell (Figure 1c). The X-ray diffraction (XRD) pattern of the hollow NPs after oxidation can be assigned both to $\gamma-\mathrm{Fe}_{2} \mathrm{O}_{3}$ and $\mathrm{Fe}_{3} \mathrm{O}_{4}$ or their mixture since these phases have very similar lattice parameters, with a trace amount of metallic iron (Figure 1d). Note that the metallic iron was still present in Mo-doped NPs even after 30 min of oxidation that pointed out to substantially higher oxidation resistance of Mo-doped NPs than undoped ones, in which no metallic iron was observed (Figure S1 in Supporting Information). No signals characteristic of molybdenum oxide $\left(\mathrm{MoO}_{2}, \mathrm{MoO}_{3}\right)$ or iron molybdate $\left(\mathrm{FeMoO}_{4}, \mathrm{Fe}_{2}\left(\mathrm{MoO}_{4}\right)_{3}\right)$ were detected. Notably, the relative intensities of the diffraction peaks from hollow NPs are significantly different from those of bulk iron oxide, indicating that the distribution of the cation vacancies in the crystalline lattice of NPs is considerably different from the bulk counterpart. ${ }^{29,30}$ Decreased (400) and increased (220) and (422) peak intensities of XRD spectra of the hollow NPs pointed out to a much higher level of cation vacancies in octahedral sites as compared to the bulk $\gamma-\mathrm{Fe}_{2} \mathrm{O}_{3}$ (for details, see the XRD simulation section and Table $\mathrm{S} 1$ and Figure S2 in Supporting Information).

In Mo-doped hollow iron oxide NPs, $\mathrm{Mo}^{6+}$ cations are in tetrahedral sites according to X-ray absorption near edge structure (XANES) analysis on Mo $L_{3}$-edge revealing the energy splitting of $\sim 2.4 \mathrm{eV}$ that is close to $2.42 \mathrm{eV}$ of $\mathrm{Na}_{2} \mathrm{MoO}_{4}$, a tetrahedrally coordinated $\mathrm{Mo}^{6+}$ compound (Figure 1e). ${ }^{31}$ Extended X-ray absorption fine structure (EXAFS) data on $\mathrm{Fe} K$-edge (Figure 1f) demonstrated much weaker signals from $\mathrm{Fe}-\mathrm{Fe}$ scattering paths in Mo-doped NPs (see Figure S3 in Supporting Information for the corresponding XANES spectra), which is characteristic of a high vacancy level of the cation $\left(\mathrm{Fe}^{3+}\right)$ sites as compared to bulk $\gamma-\mathrm{Fe}_{2} \mathrm{O}_{3}$ and undoped iron oxide NPs. In addition, increase in the concentration of vacancies in Mo-doped $(\sim 6.37 \%)$ iron oxide NPs was also confirmed by $\sim 30 \%$ higher $\mathrm{Li}^{+}$ion capacity as compared with undoped hollow iron oxide NPs (Figure 1g).

In Situ X-ray Adsorption Spectroscopy Study of MoDoped and Undoped NP Synthesis. In order to reveal the doping mechanism, we performed in situ synchrotron X-ray absorption spectroscopy measurements on the synthesis of Modoped and undoped hollow shell iron oxide NPs. The reaction was conducted using the same setup that we used for the NP synthesis in the lab. The X-ray absorption spectra were measured by irradiating X-ray beam through a slit on the reaction vessel (see Figure S4 in Supporting Information for the picture and the illustration of the setup). To monitor the formation of the NPs and the doping process in real time, we measured Fe K-edge $(7112 \mathrm{eV})$ and Mo K-edge $(20000 \mathrm{eV})$ spectra in quick scanning mode with $<50 \mathrm{~s}$ time resolution. During the measurements, the reaction mixture was heated under $\mathrm{N}_{2}$ atmosphere from $25{ }^{\circ} \mathrm{C}$ up to $180{ }^{\circ} \mathrm{C}$ and then kept at that temperature for the rest of the reaction time. Oxidation of the NPs was induced by passing dry air over the reaction mixture. Temporal changes of $\mathrm{Fe}$ and Mo K-edge XANES spectra during the synthesis of Mo-doped hollow shell iron oxide NPs are shown in Figure 2a,b, respectively. The composition of iron and molybdenum species in the reaction mixture was analyzed by linear combination fit of the data using the XANES spectra of the selected materials as the standards, and the results are plotted as functions of time in Figure 2d,e (for details, see the linear combination fit section and Figures S5-S7 in Supporting Information).

According to the fit results shown in Figure 2d, the thermal decomposition of $\mathrm{Fe}(\mathrm{CO})_{5}$ and the formation of iron NPs take place at $\sim 25 \mathrm{~min}$ of the reaction time when the temperature reaches $145{ }^{\circ} \mathrm{C}$. Prolonged heating of the reaction mixture at $180{ }^{\circ} \mathrm{C}$ leads to further decomposition of $\mathrm{Fe}(\mathrm{CO})_{5}$ and the increase in the amount of metallic iron NPs in the reaction mixture. Linear combination fit analysis assumes the presence of the small amount of iron oxide phase even before the oxidation. This can be attributed to the partial oxidation of Fe atoms on the surface of iron NPs by the residual oxygen. When about half of the $\mathrm{Fe}(\mathrm{CO})_{5}$ is converted into iron NPs, which corresponds to $144 \mathrm{~min}$ of the reaction time, we induced the oxidation by passing the air flow that led to immediate increase of the amount of iron oxide. As oxygen accelerated the decomposition of $\mathrm{Fe}(\mathrm{CO})_{5}$ by oxidizing its carbonyl group (CO) into carbon dioxide $\left(\mathrm{CO}_{2}\right),{ }^{32}$ the amount of $\mathrm{Fe}(\mathrm{CO})_{5}$ was decreased rapidly. Notably, the amount of $\gamma-\mathrm{Fe}_{2} \mathrm{O}_{3}$ is further increased for the next $5 \mathrm{~min}$ of the oxidation period.

We found that the reduction of molybdenum species $\left(\mathrm{Mo}^{6+}\right.$ $\left.\rightarrow \mathrm{Mo}^{4+}\right)$ correlates with the decomposition of $\mathrm{Fe}(\mathrm{CO})_{5}$ as well as their oxidation $\left(\mathrm{Mo}^{4+} \rightarrow \mathrm{Mo}^{6+}\right)$ with the formation of hollow iron oxide NPs (Figure 2e). In order to investigate the role of molybdenum species in the reaction kinetics, we carried out a control experiment without $\mathrm{MoO}_{2}(\mathrm{acac})_{2}$ in the reaction mixture (Figure 2c,f). Surprisingly, we found that the decomposition reaction rate of $\mathrm{Fe}(\mathrm{CO})_{5}$ in the control experiment was two times slower. For example, $45 \%$ decomposition of the initial amount of $\mathrm{Fe}(\mathrm{CO})_{5}$ occurred within $110 \mathrm{~min}$ with the molybdenum precursor versus 220 min without it. These data prove that the molybdenum precursor indeed accelerates the decomposition of $\mathrm{Fe}(\mathrm{CO})_{5}$. We assume that it takes place due to the redox reaction between $\mathrm{Mo}^{6+}$ ions of the molybdenum precursor $\left[\mathrm{MoO}_{2}(\mathrm{acac})_{2}\right]$ and $\mathrm{Fe}(\mathrm{CO})_{5}$ carbonyl groups that leads to the formation of $\mathrm{Mo}^{4+}$ and $\mathrm{CO}_{2}$, facilitating the decomposition of $\mathrm{Fe}(\mathrm{CO})_{5} \cdot{ }^{33,34}$ In addition, the presence of molybdenum species was found to lead to the higher stability of iron NPs against oxidation. In the control experiment with no molybdenum precursor, $100 \%$ oxidation of $\mathrm{Fe}(0)$ species (iron NPs and $\mathrm{Fe}(\mathrm{CO})_{5}$ ) was achieved within 35 min upon air 

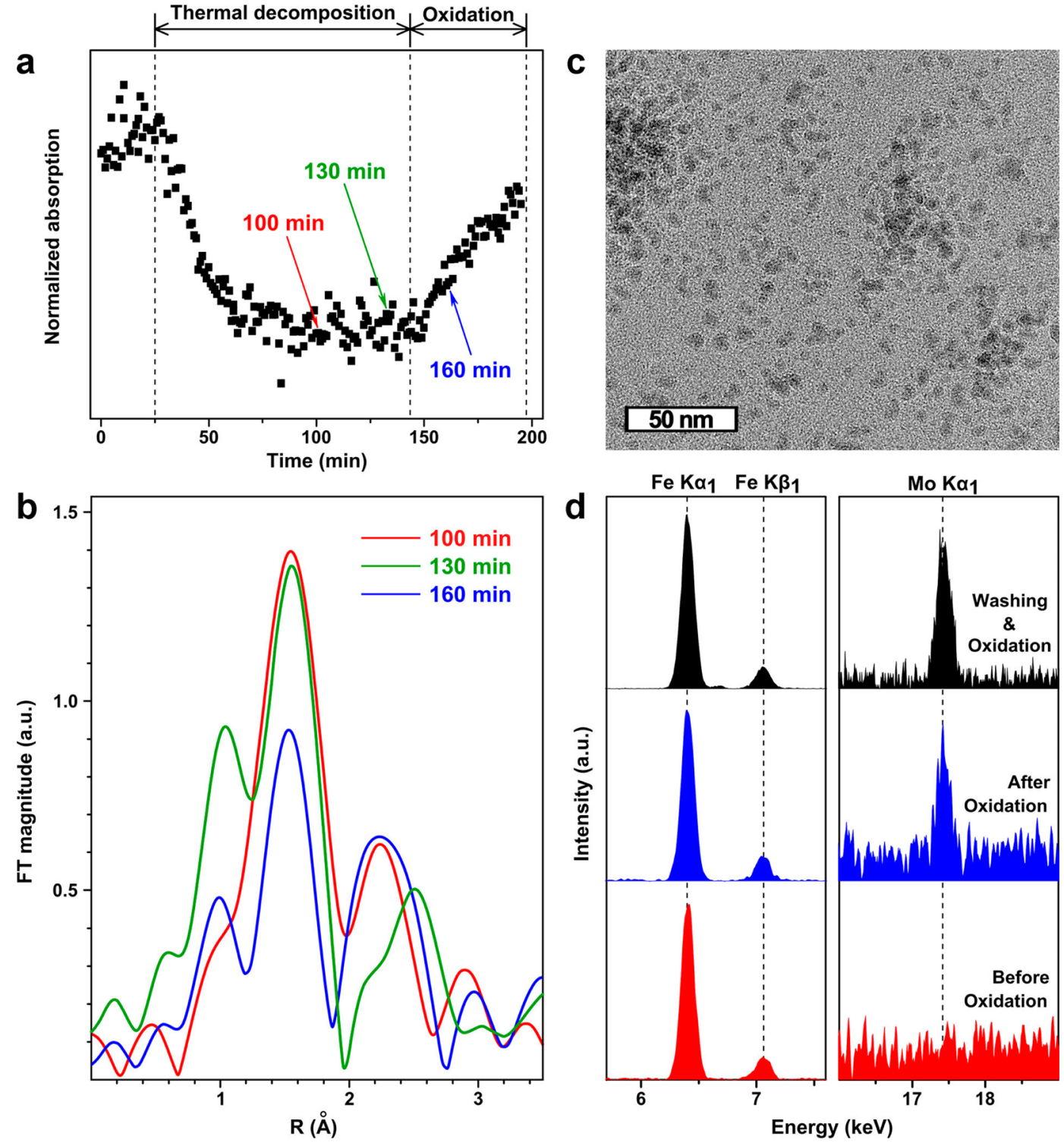

Figure 3. Changes in oxidation and coordination states of molybdenum during the synthesis of Mo-doped iron oxide NPs. (a) Temporal change in the prepeak intensity of Mo K-edge XANES spectra shown in Figure $2 \mathrm{~b}$. (b) $k^{2}$-weighted Mo EXAFS data acquired at different reaction times. (c) TEM image of iron NPs synthesized with $\mathrm{MoO}_{2}(\mathrm{acac})_{2}$ as inorganic ligand and no organic surfactant. (d) EDS spectra of thoroughly washed NPs that were sampled before (red) and after (blue) oxidation during the synthesis of Mo-doped hollow iron oxide NPs. For comparison, well-washed pure iron NPs were oxidized in a solution containing the Mo precursor and then measured by EDS (black).

exposure (Figure $2 \mathrm{f}$ ). However, only $65 \%$ of $\mathrm{Fe}(0)$ species was oxidized during the same time in the presence of the molybdenum precursor (Figure $2 \mathrm{~d}$ ). This effect is similar to the well-known anticorrosion property of molybdenum additive in steel alloys. ${ }^{35}$ Also, it was recently reported that Mo-doped metallic nanocatalysts have very good chemical stability under harsh electrochemical reaction conditions. ${ }^{36}$

Doping Mechanism. In order to study the internalization of the molybdenum atoms into the NPs we conducted in situ XANES and EXAFS measurements on Mo K-edge. The prepeak at $20007 \mathrm{eV}$ in Mo K-edge XANES spectrum is from $1 \mathrm{~s} \rightarrow 4 \mathrm{~d}$ electron transition and indicative to the coordination geometry of $\mathrm{Mo}^{6+}$ (inset of Figure $2 \mathrm{~b}$ and Figure 3a). The prepeak observed before thermal decomposition of $\mathrm{Fe}(\mathrm{CO})_{5}(0$ to $25 \mathrm{~min}$ of the reaction time) is assigned to the molybdenum precursor, $\mathrm{MoO}_{2}(\mathrm{acac})_{2}{ }^{37}$ The reduction of $\mathrm{Mo}^{6+}$ to $\mathrm{Mo}^{4+}$ during the thermal decomposition of $\mathrm{Fe}(\mathrm{CO})_{5}$ is accompanied by disappearance of the prepeak in XANES spectra since $1 \mathrm{~s} \rightarrow$ $4 \mathrm{~d}$ electron transition is not allowed for $\mathrm{Mo}^{4+}$. However, exposure of the reaction mixture to dry air led to the reemergence of the prepeak, indicating the incorporation of $\mathrm{Mo}^{6+}$ into the tetrahedral site of $\gamma-\mathrm{Fe}_{2} \mathrm{O}_{3} \mathrm{NPs}$ that is in agreement with tetrahedral coordination geometry initially revealed in ex situ molybdenum $L_{3}$-edge XANES spectrum of Mo-doped hollow iron oxide NPs (Figure 1e). Incorporation of $\mathrm{Mo}^{6+}$ into the iron oxide lattice upon oxidation is also supported by EXAFS data shown in Figure 3b. ${ }^{38,39}$ Substantial decrease in the intensity of $\mathrm{Mo}-\mathrm{O}$ correlation signal at $1.5 \AA$, which is characteristic of $\mathrm{Mo}^{6+}$ internalized in poorly crystalline oxide lattices, is observed as we switch the reaction from thermal decomposition (e.g., 100 and 130 min reaction times) to oxidation (e.g., $160 \mathrm{~min}$ reaction time).$^{38,39}$ Note, the profile of the temporal evolution of the prepeak intensities in Figure 3a resembles the shape of the plot for $\mathrm{Mo}^{6+}$ species obtained from 
linear combination fit analysis (Figure 2e). Such correlation points out that the oxidation of $\mathrm{Mo}^{4+}$ to $\mathrm{Mo}^{6+}$ and incorporation of the dopant atoms into iron oxide lattice are temporally correlated. Actually, in order to fit into cation sites of $\gamma-\mathrm{Fe}_{2} \mathrm{O}_{3}$ crystal lattice, oxidation of $\mathrm{Mo}^{4+}$ to $\mathrm{Mo}^{6+}$ is required since effective ionic radius of $\mathrm{Mo}^{4+}(0.650 \AA)$ is too large to fit in either tetrahedral or octahedral sites of $\gamma-\mathrm{Fe}_{2} \mathrm{O}_{3}{ }^{40}$ while the effective ionic radius of $\mathrm{Mo}^{6+}$ in tetrahedral site $(0.41 \AA)$ is smaller than that of $\mathrm{Fe}^{3+}$ in tetrahedral site $(0.49 \AA)$.

Fit analysis of Mo and Fe EXAFS data suggests that Mo-Fe bonding already exists even before oxidation (see the EXAFS analysis section, Tables S2-S4 and Figure S8 in Supporting Information). Thus, we conjectured that Mo species can be adsorbed on the surface of iron NPs before the oxidation. In order to confirm this, we injected $\mathrm{Fe}(\mathrm{CO})_{5}$ into a solution containing only $\mathrm{MoO}_{2}(\mathrm{acac})_{2}$ and no organic surfactant at 180 ${ }^{\circ} \mathrm{C}$. This reaction yielded iron NPs (Figure 3c) indicating that molybdenum species can indeed serve as inorganic ligand for the iron NPs. Such Fe NPs have good colloidal stability only at elevated temperature and cooling the reaction mixture to room temperature results in the partial precipitation of $\mathrm{Fe}$ NPs stabilized with molybdenum species. Nevertheless, they can form a stable colloidal solution after adding a small amount of oleylamine.

The binding ability of molybdenum species seems to be not strong enough since repeated washing of the NPs with acetone can completely remove molybdenum from the surface of iron NPs. For example if we take an aliquot of Fe NPs synthesized in the presence of $\mathrm{MoO}_{2}(\mathrm{acac})_{2}$ and thoroughly wash them with acetone, no Mo signal is detected by energy-dispersive $\mathrm{X}$ ray spectroscopy (EDS) (Figure 3d). However, in oxidized NPs the atomic ratio of $\mathrm{Fe}$ and Mo was found to be $\sim 100: 6.37$. To summarize, data shown in Figure 3 confirm two facts: (i) molybdenum species are chemically adsorbed on the surface of iron NPs and no molybdenum is present in metallic iron NPs; and (ii) molybdenum ions are internalized into the lattice of the NPs only upon oxidation.

The previously reported strategies of direct synthesis of doped NPs are based either on trapping or diffusion doping mechanism. ${ }^{5,16-23}$ In order to validate the applicability of these well-known mechanisms we conducted a number of controlled experiments.

First, since a significant amount of $\mathrm{Fe}(\mathrm{CO})_{5}$ remains in the solution before oxidation (Figure 2d), we have to exclude that the molybdenum doping can occur via trapping of molybdenum cations adsorbed at the surface of the NPs by the deposition of iron oxide shell from remaining $\mathrm{Fe}(\mathrm{CO})_{5}$. To investigate the possibility of trapping mechanism, we prepared iron NPs with no $\mathrm{MoO}_{2}(\mathrm{acac})_{2}$ and washed them thoroughly from the residual $\mathrm{Fe}(\mathrm{CO})_{5}$. After that iron NPs were redispersed in the octadecene solution containing only molybdenum precursor and oxidized them by bubbling dry air at $180{ }^{\circ} \mathrm{C}$ for $40 \mathrm{~min}$. The ratio of Fe to Mo in oxidized NPs was $\sim 100: 6$ as measured by EDS (Figure 3d). Similar to Modoped NPs prepared according to our standard method, these doped iron oxide NPs prepared via surface modification followed by oxidation also showed substantial increase of the $\mathrm{Li}^{+}$ion capacity in electrochemical tests (Figure $1 \mathrm{~g}$ ). These results rule out the possibility of the trapping as the process responsible for the internalization of molybdenum in hollow iron oxide NPs. Based on numerous experiment we assume that the unreacted $\mathrm{Fe}(\mathrm{CO})_{5}$ upon oxidation is used for nucleation and growth of small (often less than $1.5 \mathrm{~nm}$ ) iron oxide NPs that are typically discarded in a standard washing procedure

Second, in order to evaluate the possibility of doping as a result of Mo diffusion into vacant iron oxide lattice we conducted two control experiments: The diffusion of Mo ions from the solution into the NPs was tested by (i) prolonged oxidation of already oxidized NPs prepared without $\mathrm{MoO}_{2}(\mathrm{acac})_{2}$ and redispersed in a solution containing molybdenum precursors; and (ii) prolonged annealing ( $\sim 2$ h) of already oxidized Mo-doped hollow iron oxide NPs at 180 ${ }^{\circ} \mathrm{C}$ in the presence of molybdenum species. In the first control experiment the doping (if any) was below the detection limit of our EDS system. In the second experiment we found that prolonged annealing did not affect the Mo doping level of the hollow iron oxide NPs. These results confirm that the ion diffusion through oxide lattice of NPs has no significant contribution into the doping process.

Based on the results from in situ XANES, EXAFS, and our controlled experiments, we derived a new doping mechanism that takes place during the synthesis of Mo-doped hollow shell iron oxide NPs (Figure 4). The decomposition of $\mathrm{Fe}(\mathrm{CO})_{5}$

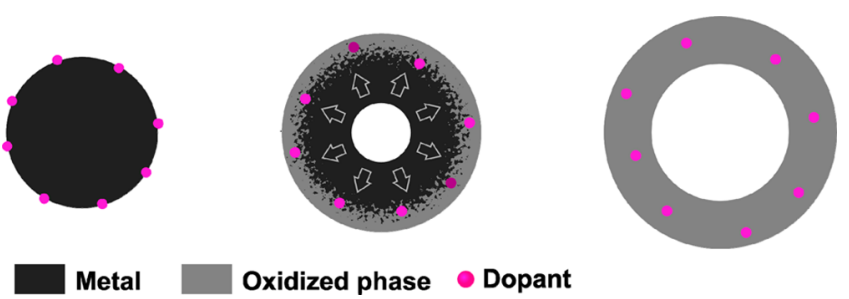

Figure 4. Depiction of the oxidation induced doping mechanism. A metallic NP with dopant ions adsorbed on the surface (left). Early stage of the oxidation of metallic NP indicating the capturing of dopant ions inside of the oxidized layer (middle); doped hollow shell NP after full oxidation (right).

leads to the formation of iron NPs with $\mathrm{Mo}^{4+}$ ions adsorbed at their surface. After the air is introduced, metallic iron NPs are transformed to iron oxide hollow shell NPs by the Kirkendall effect. ${ }^{27,28}$ In this effect, oxidation of metal ions $\left(\mathrm{Fe}^{0} \rightarrow \mathrm{Fe}^{2+/ 3+}\right.$ and $\left.\mathrm{Mo}^{4+} \rightarrow \mathrm{Mo}^{6+}\right)$ induces the outward mass transport from the core of the NPs. As the structure of the NP is changed from metallic iron to hollow shell iron oxide, $\mathrm{Mo}^{6+}$ ions migrate into the cation sites of the iron oxide lattice. It is notable that in this process the mass transport of the host materials ( $\mathrm{Fe}$ and $\mathrm{O}$ ), and not the diffusion of the dopant, is responsible for doping. This differentiates the oxidation induced doping mechanism from the other previously reported doping mechanisms. In the trapping mechanism, both the lattice of the core and the dopant atoms are stationary during the further growth of the shell on them. ${ }^{22,23}$ Doping by ionic diffusion requires the dopant ions move through the stationary host lattice of NPs. ${ }^{24,25}$ However, in our mechanism the dopant that was once at the outside surface the NPs is internalized by the outward flow of the host material. This unique process contributes to fast (within few minutes) and effective doping of NPs through deliberate oxidation.

The oxidation induced doping allows controlling the doping levels simply by varying the amount of dopant precursor. Complete oxidation of preformed $\sim 11 \mathrm{~nm}$ iron NPs in the presence of $0.05,0.075$, and $0.1 \mathrm{~g}$ of $\mathrm{MoO}_{2}(\mathrm{acac})_{2}$ resulted in the doping levels of 1.68 at \%, 6.37 at \%, and 8.4 at \%, 
respectively (for detail, see Figure S9 in Supporting Information).

Cobalt-Based NP Systems. Different oxidized forms of transition metals (e.g., oxides, dichalcogenides, and phosphosulfides) have been recently found to be very appealing to design cost-effective electrocatalysts that can potentially replace iridium- and platinum-based catalysts. ${ }^{41-43}$ Since the doping is known to modulate the electrochemical performance of electrocatalysts we investigated the applicability of our doping mechanism to the other NP systems that can be of interest for energy conversion and storage applications. We conducted study on cobalt-based NP systems since the synthesis of cobalt $\mathrm{NPs}$ is well established. We found that Mo precursor $\left[\mathrm{MoO}_{2}(\mathrm{acac})_{2}\right]$ demonstrated the same effects in the synthesis of $\varepsilon$-Co NPs. ${ }^{44}$ As in the case of iron NPs, we observed high oxidation resistance of $\varepsilon$-Co NPs. According to XRD data, cobalt NPs synthesized without the molybdenum precursor are fully oxidized into $\mathrm{CoO}$ within $1 \mathrm{~h}$ upon exposure to the ambient atmosphere. However, the NPs synthesized in the presence of the Mo precursor in the reaction mixture are much more stable against oxidation as evidenced by dominant $\varepsilon$-Co phase in the XRD pattern of the NPs measured after 2 weeks of the exposure to air under ambient conditions (Figure 5a). Co $K$-edge XANES data show that the content of $\mathrm{CoO}$ phase in $\varepsilon$ Co NPs is less than 20\% (Figure S10 in Supporting Information). Subsequent oxidation of as-prepared Momodified $\varepsilon$-Co NPs (Figure $5 b$ ) by bubbling air or injecting sulfur solution at $180{ }^{\circ} \mathrm{C}$ results in the formation of $\mathrm{Co} / \mathrm{CoO}$ and cobalt sulfide NPs (Figures 5c,d). EDS analysis indicates that the concentration of $\mathrm{Mo}$ in $\mathrm{Co} / \mathrm{CoO}$ and $\mathrm{Co}_{3} \mathrm{~S}_{4} \mathrm{NPs}$ are 0.54 at $\%$ and 1.35 at \%, respectively. Similar to iron NPs, cobalt NPs can be synthesized without organic surfactants but only in the presence of Mo precursor (Figure 5e).

In conclusion, we conducted for the first time real-time in situ XANES/EXAFS study of the formation kinetics of Mo-doped and undoped iron oxide NPs. Our study revealed a new oxidation induced doping mechanism by which the mass flow of the host material triggered by oxidation can lead to fast and efficient doping of the NPs, and the doping level is controlled by varying the amount of the dopant precursor. The general applicability of the doping mechanism discovered for iron oxide NPs was validated for oxidized forms of cobalt (oxide and sulfide). Importantly, our in situ measurement technique is generally applicable for time-resolved mechanism study of various doped NPs synthesis using the conventional reaction setup that we use in the laboratory. Moreover, time-resolved XANES/EXAFS study revealed that the reaction kinetics of the nucleation, growth, and oxidation of the host NPs are significantly affected by the dopant precursor. We also demonstrate proof of principle that NPs can be synthesized in organic solutions with only inorganic capping ligands. In addition, we found that both metallic iron and cobalt NPs synthesized in the presence of molybdenum precursor show strong resistance toward oxidation. We believe that oxidation induced doping mechanism can be applied to a number of the other NP systems where the mass transport can be induced chemically (e.g., oxidation) or electrochemically.

Experimental Details. Nanoparticle Synthesis. In a typical synthesis, a reaction mixture containing $0.075 \mathrm{~g}$ of $\mathrm{MoO}_{2}(\mathrm{acac})_{2}$ and $0.1 \mathrm{~g}$ of oleylamine in $10 \mathrm{~mL}$ of 1 -octadecene (ODE) was prepared and heated up to $180{ }^{\circ} \mathrm{C}$ under $\mathrm{N}_{2}$ into which $0.6 \mathrm{~g}$ of $\mathrm{Fe}(\mathrm{CO})_{5}$ was rapidly injected. After injection, the mixture was left at the same temperature for $30 \mathrm{~min}$ for full
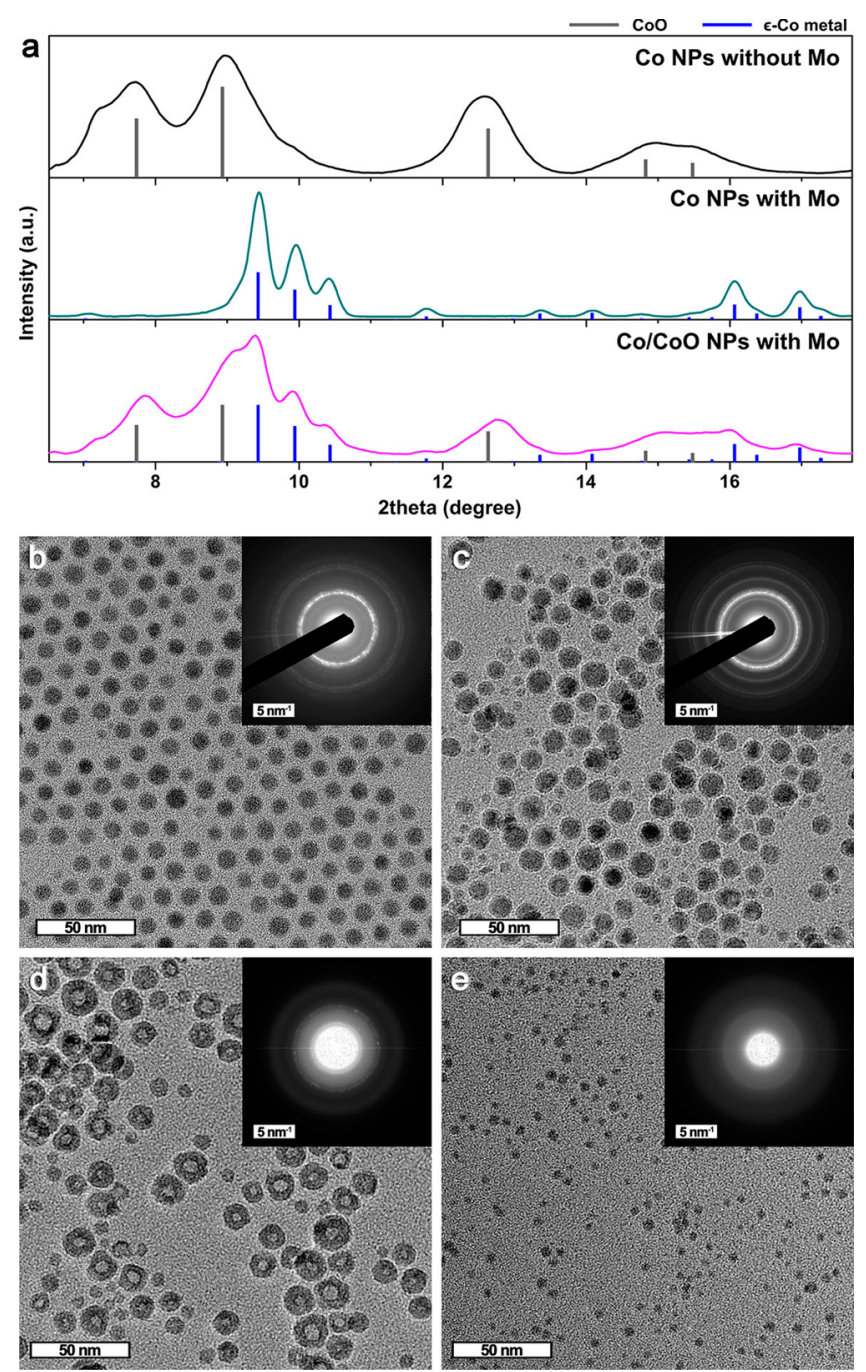

Figure 5. Characterization of cobalt and cobalt-based NPs. (a) XRD patterns of cobalt NPs synthesized without and with the Mo precursor and stored under ambient conditions. In the bottom, XRD pattern of $\mathrm{Co} / \mathrm{CoO}$ NPs prepared by bubbling the reaction mixture with air. (be) TEM images and electron diffraction patterns of $\varepsilon$-Co NPs synthesized in the presence of the Mo precursor (b), $\mathrm{Co} / \mathrm{CoO}$ NPs (c), cobalt sulfide NPs (d), and $\varepsilon$-Co NPs synthesized without organic surfactants (e).

decomposition of $\mathrm{Fe}(\mathrm{CO})_{5}$. Then dry air is bubbled into the mixture through a syringe needle for $1 \mathrm{~min}$ at the flow rate of $\sim 100 \mathrm{~mL} / \mathrm{min}$, while keeping the temperature at $180^{\circ} \mathrm{C}$. The molar ratio of $\mathrm{Fe} / \mathrm{Mo} /$ amine is 100:7.5:12 in the mixture, and the synthesis was conducted by using the standard Schlenk technique. The NPs obtained were repeatedly washed with acetone and redispersed in toluene. Iron NPs before oxidation were obtained by drawing an aliquot of the reaction mixture before bubbling air and separating NPs from the aliquots with excess acetone. Undoped iron oxide hollow NPs were synthesized following the same procedure but with no $\mathrm{MoO}_{2}(\mathrm{acac})_{2}$ in the mixture.

For the synthesis of Mo-doped cobalt oxide NPs, a reaction mixture of $0.09 \mathrm{~g}$ of oleic acid, $0.05 \mathrm{~g}$ of trioctylphosphine oxide, and $0.05 \mathrm{~g}$ of $\mathrm{MoO}_{2}(\mathrm{acac})_{2}$ in $12 \mathrm{~mL}$ of 1,2dichlorobenzene (DCB) and a cobalt stock solution of $0.27 \mathrm{~g}$ $\mathrm{Co}_{2}(\mathrm{CO})_{8}$ in $1.5 \mathrm{~mL}$ of $\mathrm{DCB}$ were prepared, separately. The reaction mixture was heated up to $180{ }^{\circ} \mathrm{C}$ under $\mathrm{N}_{2}$, and then 
the stock solution was rapidly injected into it. The mixture was kept at $180{ }^{\circ} \mathrm{C}$ for $45 \mathrm{~min}$ before bubbling dry air. Mo-doped and undoped cobalt NPs were obtained by stopping the reaction before bubbling air. Mo-doped cobalt sulfide NPs were synthesized by adding a sulfur stock solution of $0.05 \mathrm{~g}$ of elemental sulfur in $0.5 \mathrm{~g}$ of DCB instead of air bubbling, and the rest of the procedure is the same as the synthesis of Modoped cobalt oxide NPs.

In Situ Synchrotron X-ray Absorption Spectroscopy Measurements. For the in situ measurements, the synthetic method of Mo-doped iron oxide hollow NPs was modified in order to slow down the overall reaction rate with respect to the scan time of the XAS measurement setup. In this method, a reaction mixture of $0.11 \mathrm{~g}$ of $\mathrm{MoO}_{2}(\mathrm{acac})_{2}, 0.17 \mathrm{~g}$ of oleylamine, and $1.73 \mathrm{~g}$ of $\mathrm{Fe}(\mathrm{CO})_{5}$ in $28 \mathrm{~mL}$ of ODE was prepared. The molar ratio of $\mathrm{Fe} / \mathrm{Mo} /$ amine is $100: 4: 7$, and the absolute concentration of $\mathrm{Fe}$ is the same as the typical synthesis. During the measurement, the mixture was heated from room temperature up to $180{ }^{\circ} \mathrm{C}$ at the rate of $3 \mathrm{~K} / \mathrm{min}$ under $\mathrm{N}_{2}$ and kept at that temperature for a certain period of time $(2-4 \mathrm{~h})$ for the formation of iron NPs. After then, the NPs were oxidized at $180{ }^{\circ} \mathrm{C}$ by introducing dry air into the reaction vessel through a syringe needle at the flow rate of 50 $\mathrm{mL} / \mathrm{min}$ using a remote gas flow controller. The needle tip was place well above the surface of the reaction mixture to prevent bubbling. The synthesis was conducted using the same equipment including the Schlenk line and the glassware as those used in the synthesis lab except for the reaction vessel. On the one side of the reaction flask, there was a slit of $2 \mathrm{~cm} \times$ $0.5 \mathrm{~cm}$ in size that is covered with polyimide film and sealed with epoxy resin, and the X-ray beam was irradiated to the reaction mixture through the slit during the reaction. The $\mathrm{X}$-ray absorption signal from the solution is measured in fluorescence mode by a detector placed in 90-degree position with respect to the incident beam.

In situ X-ray absorption spectroscopy measurements on $\mathrm{Fe}$ K-edge $(7112 \mathrm{eV})$ and Mo K-edge $(20000 \mathrm{eV})$ for the samples were measured at 10-ID-B beamline at the Advanced Photon Source, Argonne National Laboratory. Rhodium-coated harmonic rejection mirror was used to eliminate higher energy photons. Experiments were performed in fluorescence mode using ion chamber with Stern Heald geometry. For Fe edge, a mixture of $80 \% \mathrm{He}$ and $20 \% \mathrm{~N}_{2}$ gas was used in the initial ion chamber $I_{0}$ placed before the sample and full $\mathrm{N}_{2}$ gas in transmission and reference ion chambers. Ar gas was used for fluorescence ion chamber. For Mo edge, pure $\mathrm{N}_{2}$ was used in the $I_{0}$ ion chamber and a mixture of $50 \% \mathrm{~N}_{2}$ and $50 \%$ Ar gas in the transmission and reference ion chambers. $\mathrm{Kr}$ gas was used in the fluorescence ion chamber. The reaction vessel was put on the sample stage so that the incident angle of the X-ray beam with respect to the surface of the slit of the vessel is $45 \mathrm{deg}$. Beamline was calibrated to $7112 \mathrm{eV}$ with Fe metal foil and later to $20000 \mathrm{eV}$ with Mo metal foil before $\mathrm{Fe}$ and Mo edge measurements, respectively. Iron standards and Mo standards were measured in transmission geometry with Fe or Mo foil, respectively, in front of the reference ion chamber. During the in situ measurements, $\mathrm{Fe}$ or Mo foil was measured with a photodiode for every scan taken to check for any energy shift during the measurements. The spot size of the incident X-ray beam on the sample was $800 \mu \mathrm{m} \times 800 \mu \mathrm{m}$. Measurements were done in quick scanning mode, where undulator gap and taper were fixed for each of the edges, while Bragg angle of $\mathrm{Si}(111)$ double crystal monochromator was scanned with constant speed. The XAS scans were taken from 6900 to 7900 $\mathrm{eV}$ for Fe edge and from 19800 to $20640 \mathrm{eV}$ for Mo edge. In both scans, the scan rate was $0.015 \mathrm{~s}$ per step with the step size of $0.4 \mathrm{eV}$, and each scan took $\sim 40 \mathrm{~s}$ for Fe edge and $\sim 30 \mathrm{~s}$ for Mo edge. The scans were taken continuously until the completion of the experiments.

Data processing and analysis were performed using ATHENA software. ${ }^{45}$ Linear combination fit of each spectrum was calculated in the range between $E_{0}-20 \mathrm{eV}$ and $E_{0}+80 \mathrm{eV}$, and the weights sum of the fit was constrained to be 1 . The EXAFS oscillations $\chi(k)$ were extracted from the data as a function of photoelectron wavenumber $k$ following standard procedures. The theoretical paths were generated using FEFF6, and the modeling was done in the conventional way using ARTEMIS with FEFF6 built into it. ${ }^{46,47}$ Fitting parameters were obtained by modeling the EXAFS data of each sample in $R$-space until a satisfactory fit describing the system was obtained. Data sets were simultaneously fitted in $R$-space with $k$-weights of 1,2 , and 3. Amplitude $\left(S_{\mathrm{o}}{ }^{2}\right)$ for each of the elements was obtained from fitting their respective metal foils.

Characterization. Ex situ synchrotron X-ray absorption spectroscopy on Mo $L_{3}$-edge $(2520 \mathrm{eV})$ and Fe $K$-edge (7112 $\mathrm{eV}$ ) for the samples were measured at the SXS beamline and the XAFS-2 beamline, respectively, at the Laboratorio Nacional de Luz Sincrotron (LNLS, Campinas, SP, Brazil). For Mo $L_{3}$ edge measurement, an $\operatorname{InSb}(111)$ double crystal monochromator with a slit aperture of $1.0 \mathrm{~mm}$ was used to obtain a resolution about $0.7 \mathrm{eV}$. The X-ray absorption spectra were recorded by fluorescence mode. Experiments were performed in a vacuum of $10^{-7} \mathrm{mbar}$ at room temperature. The energy scale was calibrated to Mo $L_{3}$-edge of Mo metallic foil. Fe $K$ edge spectra were recorded at room temperature using a $\mathrm{Si}(111)$ double crystal monochromator in transmission mode with three ion chambers as detectors. The absorption of the sample was determined between the first two chambers, and the third one was used to measure the signal from Fe metal foil placed between the last two chambers as reference. The data were processed and analyzed by standard methods using ATHENA software. ${ }^{45}$

For electrochemical tests, the NPs electrodes were fabricated following the previously reported method from our group. ${ }^{48}$ The NPs electrodes were assembled into 2032 coin type cells using a $\mathrm{Li}$ metal foil as the counter electrode for lithium halfcell tests. Microporous polyolefin separators (Celgard 2325) and 1.2 $\mathrm{M} \mathrm{LiPF}_{6}$ in ethylene carbonate/ethyl methyl carbonate (3:7 weight ratio) electrolyte (Tomiyama) were used in the cells. Half-cell cycles were operated at $300 \mathrm{~mA} / \mathrm{g}$ between 4.5 and $1.5 \mathrm{~V}$ vs $\mathrm{Li} / \mathrm{Li}^{+}$using an automated Maccor battery tester at ambient temperature. All cell assembly operations were performed in a He-filled dry glovebox (oxygen level $<2 \mathrm{ppm}$ ).

Synchrotron X-ray diffraction (XRD) measurements were performed at GSECARS Sector 13-ID-D at the Advanced Photon Source, Argonne National Laboratory. The photon energy was $40 \mathrm{keV}(0.3100 \AA)$ for the iron samples (Figure 1d) and $37 \mathrm{keV}(0.3344 \AA)$ for cobalt samples (Figure 5a). Transmission electron microscopy (TEM), energy-filtered TEM, and energy dispersive X-ray spectroscopy (EDS) data were obtained by using a Jeol JEM-2100F equipped with a Gatan GIF Quantum Energy Filters and an Oxford X-Max ${ }^{\mathrm{N}} 80$ TLE detector and operated at $200 \mathrm{kV}$. 


\section{ASSOCIATED CONTENT}

\section{S Supporting Information}

The Supporting Information is available free of charge on the ACS Publications website at DOI: 10.1021/acs.nanolett.6b01072.

Schematic illustration of synthetic protocol of Mo-doped iron oxide hollow shell NPs, XRD patterns of undoped iron/iron oxide NPs before and after oxidation, simulated XRD patterns of $\gamma-\mathrm{Fe}_{2} \mathrm{O}_{3}$ with various vacancy levels, $\mathrm{Fe}$ $K$-edge XANES spectra of bulk $\gamma-\mathrm{Fe}_{2} \mathrm{O}_{3}$, undoped and Mo-doped NPs, photos of the setup for in situ X-ray absorption spectroscopy measurement, $\mathrm{Fe}$ and Mo Kedge XANES spectra of various standard materials, additional linear combination fit data of $\mathrm{Fe} K$-edge data from Figure $2 \mathrm{a}$ and Mo K-edge data from Figure $2 \mathrm{~b}$, EXAFS fit results of the data in Figures 2a,b, TEM images of Mo-doped hollow shell iron oxide NPs with various doping levels and plot for doping level vs the amount of Mo precursor, and Co K-edge XANES data of Co NPs, bulk CoO, and cobalt metal (PDF)

\section{AUTHOR INFORMATION}

\section{Corresponding Author}

*E-mail: eshevchenko@anl.gov.

\section{Present Addresses}

(S.G.K.) Center for Nanoparticle Research, Institute for Basic Science \& Seoul National University, Seoul 151-742, Republic of Korea.

(S.C.) Physical Sciences Department, Elgin Community College, 1700 Spartan Drive, Elgin, IL 60123, United States.

(B.K.) Heliotrope Technologies, 2625 Alcatraz Ave \#377, Berkeley, CA 94705, United States.

(T.S.) Materials Analysis \& Global Materials Science, Kennametal Inc. 1600 Technology Way, Latrobe, PA 15650, United States.

\section{Notes}

The submitted manuscript has been created by UChicago Argonne, LLC, Operator of Argonne National Laboratory ("Argonne"). Argonne, a U.S. Department of Energy Office of Science laboratory, is operated under Contract No. DE-AC0206CH11357. The U.S. Government retains for itself, and others acting on its behalf, a paid-up nonexclusive, irrevocable worldwide license in said article to reproduce, prepare derivative works, distribute copies to the public, and perform publicly and display publicly, by or on behalf of the Government.

The authors declare no competing financial interest.

\section{ACKNOWLEDGMENTS}

Use of the Center for Nanoscale Materials and Advanced Photon Source was supported by the U.S. Department of Energy, Office of Science, Office of Basic Energy Sciences, under Contract No. DE-AC02-06CH11357. MRCAT is funded by MRCAT host institutions. The work at GeoSoilEnviroCARS was supported by the National Science Foundation-Earth Sciences (EAR-0622171) and Department of Energy-Geosciences (DE-FG02-94ER14466). The Mo L-edge and Fe Kedge XAFS ex situ synchrotron experiments were performed at SXS and XAFS-2 beamlines, respectively (LNLS, Campinas). F.G.R. and L.J.G. acknowledge CONICET, Argentina (grant \# PIP-1035). We acknowledge Prof. Jeffrey T. Miller (Purdue
University) for helpful discussion on the in situ XAS experiments and Prof. Carlo U. Segre (Illinois Institute of Technology) for allocation of staff beam time.

\section{REFERENCES}

(1) Erwin, S. C.; Zu, L.; Haftel, M. I.; Efros, A. L.; Kennedy, T. A.; Norris, D. J. Nature 2005, 436, 91-94.

(2) Mocatta, D.; Cohen, G.; Schattner, J.; Millo, O.; Rabani, E.; Banin, U. Science 2011, 332, 77-81.

(3) Bryan, J. D.; Gamelin, D. R. Prog. Inorg. Chem. 2005, 54, 47-126.

(4) Yu, J. H.; Kwon, S. H.; Petrášek, Z.; Park, O. K.; Jun, S. W.; Shin, K.; Choi, M.; Park, Y. I.; Park, K.; Na, H. B.; Lee, N.; Lee, D. W.; Kim, J. H.; Schwille, P.; Hyeon, T. Nat. Mater. 2013, 12, 359-366.

(5) Bradshaw, L. R.; May, J. W.; Dempsey, J. L.; Li, X.; Gamelin, D. R. Phys. Rev. B: Condens. Matter Mater. Phys. 2014, 89, 115312.

(6) Yang, J.; Fainblat, R.; Kwon, S. G.; Muckel, F.; Yu, J. H.; Terlinden, H.; Kim, B. H.; Iavarone, D.; Choi, M. K.; Kim, I. Y.; Park, I.; Hong, H.-K.; Lee, J.; Son, J. S.; Lee, Z.; Kang, K.; Hwang, S.-J.; Bacher, G.; Hyeon, T. J. Am. Chem. Soc. 2015, 137, 12776-12779.

(7) Wang, F.; Han, Y.; Lim, C. S.; Lu, Y.; Wang, J.; Xu, J.; Chen, H.; Zhang, C.; Hong, M.; Liu, X. Nature 2010, 463, 1061-1065.

(8) Gargas, D. J.; Chan, E. M.; Ostrowski, A. D.; Aloni, S.; Altoe, M. V. P.; Barnard, E. S.; Sanii, B.; Urban, J. J.; Milliron, D. J.; Cohen, B. E.; Schuck, P. J. Nat. Nanotechnol. 2014, 9, 300-305.

(9) Guo, H.; Song, X.; Zhuo, Z.; Hu, J.; Liu, T.; Duan, Y.; Zheng, J.; Chen, Z.; Yang, W.; Amine, K.; Pan, F. Nano Lett. 2016, 16, 601-608.

(10) Zhang, L.; Wu, H. B.; Lou, X. W. Adv. Energy Mater. 2014, 4, 1300958.

(11) Hahn, B. P.; Long, J. W.; Mansour, A. N.; Pettigrew, K. A.; Osofsky, M. S.; Rolison, D. R. Energy Environ. Sci. 2011, 4, 1495-1502.

(12) Hahn, B. P.; Long, J. W.; Rolison, D. R. Acc. Chem. Res. 2013, 46, 1181-1191.

(13) Norris, D. J.; Efros, A. L.; Erwin, S. C. Science 2008, 319, 17761779.

(14) Dalpian, G. M.; Chelikowsky, J. R. Phys. Rev. Lett. 2006, 96, 226802.

(15) Yang, Y.; Chen, O.; Angerhofer, A.; Cao, Y. C. J. Am. Chem. Soc. 2008, 130, 15649-15661.

(16) Yang, Y.; Chen, O.; Angerhofer, A.; Cao, Y. C. J. Am. Chem. Soc. 2006, 128, 12428-12429.

(17) Whitham, P. J.; Knowles, K. E.; Reid, P. J.; Gamelin, D. R. Nano Lett. 2015, 15, 4045-4051.

(18) Fainblat, R.; Muckel, F.; Barrows, C. J.; Vlaskin, V. A.; Gamelin, D. R.; Bacher, G. ACS Nano 2014, 8, 12669-12675.

(19) Zhao, Y.; Rabouw, F. T.; Puffelen, T. v.; Walree, C. A. v.; Gamelin, D. R.; Donegá, C. d. M.; Meijerink, A. J. Am. Chem. Soc. 2014, 136, 16533-16543.

(20) Li, X.; Wang, R.; Zhang, F.; Zhao, D. Nano Lett. 2014, 14, 3634-3639.

(21) Buonsanti, R.; Milliron, D. J. Chem. Mater. 2013, 25, 13051317.

(22) Du, M. H.; Erwin, S. C.; Efros, A. L. Nano Lett. 2008, 8, 28782882.

(23) Viswanatha, R.; Brovelli, S.; Pandey, A.; Crooker, S. A.; Klimov, V. I. Nano Lett. 2011, 11, 4753-4758.

(24) Vlaskin, V. A.; Barrows, C. J.; Erickson, C. S.; Gamelin, D. R. J. Am. Chem. Soc. 2013, 135, 14380-14389.

(25) Sahu, A.; Kang, M. S.; Kompch, A.; Notthoff, C.; Wills, A. W.; Deng, D.; Winterer, M.; Frisbie, C. D.; Norris, D. J. Nano Lett. 2012, $12,2587-2594$.

(26) Rivest, J. B.; Jain, P. K. Chem. Soc. Rev. 2013, 42, 89-96.

(27) Koo, B.; Xiong, H.; Slater, M. D.; Prakapenka, V. B.; Balasubramanian, M.; Podsiadlo, P.; Johnson, C. S.; Rajh, T.; Shevchenko, E. V. Nano Lett. 2012, 12, 2429-2435.

(28) Cabot, A.; Puntes, V. F.; Shevchenko, E. V.; Yin, Y.; Balcells, L.; Marcus, M. A.; Hughes, S. M.; Alivisatos, A. P. J. Am. Chem. Soc. 2007, 129, 10358-10360. 
(29) Korobeinikova, A. V.; Fedeeva, V. I.; Reznitskii, L. A. J. Struct. Chem. 1976, 17, 737-741.

(30) Greaves, C. J. Solid State Chem. 1983, 49, 325-333.

(31) Lede, E. J.; Requejo, F. G.; Pawelec, B.; Fierro, J. L. G. J. Phys. Chem. B 2002, 106, 7824-7831.

(32) Crabtree, R. H. The Organometallic Chemistry of the Transition Metals, 4th ed.; John Wiley \& Sons, Inc.: Hoboken, NJ, 2005.

(33) El-Shamy, H. K.; El-Aggan, A. M. J. Am. Chem. Soc. 1953, 75, 1187-1190.

(34) Williams, C. C.; Ekerdt, J. G. J. Phys. Chem. 1993, 97, 68436852.

(35) Clayton, C. R.; Lu, Y. C. J. Electrochem. Soc. 1986, 133, 24652473.

(36) Huang, X.; Zhao, Z.; Cao, L.; Chen, Y.; Zhu, E.; Lin, Z.; Li, M.; Yan, A.; Zettl, A.; Wang, Y. M.; Duan, X.; Mueller, T.; Huang, Y. Science 2015, 348, 1230-1234.

(37) Aritani, H.; Tanaka, T.; Funabiki, T.; Yoshida, S.; Kudo, M.; Hasegawa, S. J. Phys. Chem. 1996, 100, 5440-5446.

(38) Stern, E. A. Contemp. Phys. 1978, 19, 239-310.

(39) Kuzmin, A.; Purans, J. J. Phys.: Condens. Matter 2000, 12, 19591970.

(40) Shannon, R. D. Acta Crystallogr., Sect. A: Cryst. Phys., Diffr., Theor. Gen. Crystallogr. 1976, A32, 751-767.

(41) Wang, D.-Y.; Gong, M.; Chou, H.-L.; Pan, C.-J.; Chen, H.-A.; Wu, Y.; Lin, M.-C.; Guan, M.; Yang, J.; Chen, C.-W.; Wang, Y.-L.; Hwang, B.-J.; Chen, C.-C.; Dai, H. J. Am. Chem. Soc. 2015, 137, 15871592.

(42) Chernev, P.; Zaharieva, I.; Sicklinger, J.; Stefanic, G.; Döblinger, M.; Müller, A.; Pokharel, A.; Böcklein, S.; Scheu, C.; Bein, T.; Fattakhova-Rohlfing, D.; Fominykh, K. ACS Nano 2015, 9, 51805188.

(43) Cabán-Acevedo, M.; Stone, M. L.; Schmidt, J. R.; Thomas, J. G.; Ding, Q.; Chang, H.-C.; Tsai, M.-L.; He, J.-H.; Jin, S. Nat. Mater. 2015, 14, 1245-1251.

(44) Dinega, D. P.; Bawendi, M. G. Angew. Chem., Int. Ed. 1999, 38, $1788-1791$.

(45) Newville, M. J. Synchrotron Radiat. 2001, 8, 322-324.

(46) Rehr, J. J.; Albers, R. C. Rev. Mod. Phys. 2000, 72, 621-654.

(47) Ravel, B.; Newville, M. J. Synchrotron Radiat. 2005, 12, 537541.

(48) Koo, B.; Goli, P.; Sumant, A. V.; Claro, P. C. d. S.; Rajh, T.; Johnson, C. S.; Balandin, A. A.; Shevchenko, E. V. ACS Nano 2014, 8, $7202-7207$. 